\title{
Magnetization profile of ultrathin FePd films
}

\author{
H. A. Dürr, ${ }^{a \star} \dagger$ E. Dudzik, ${ }^{a, b}$ S. S. Dhesi, ${ }^{a}$ J. B. Goedkoop, ${ }^{c}$ G. van der Laan, ${ }^{a}$ M. \\ Belakhovsky, ${ }^{d}$ C. Mocuta, ${ }^{d}$ A. Marty ${ }^{d}$ and Y. Samson ${ }^{d}$ \\ ${ }^{a}$ Magnetic Spectroscopy Group, Daresbury Laboratory, Warrington WA4 4AD, UK, \\ ${ }^{b}$ Department of Physics, University of York, York YO1 5DD, UK, ${ }^{C}$ University of Amsterdam, \\ Valckenierstraat 65, NL 1018 XE Amsterdam, The Netherlands, and ${ }^{d}$ CEA/Grenoble, Service \\ de Physique des Matériaux et Microstructures, 17 rue des Martyrs, 38054 Grenoble CEDEX \\ 9, France.E-mail: h.durr@fz-juelich.de
}

(Received 10 September 1999; accepted 8 November 1999)

\begin{abstract}
The method of circular dichroism in X-ray resonant magnetic scattering is presented which allows a straightforward determination of the magnetization profile of magnetic patterns in ultrathin films. Application to single crystalline FePd layers shows unambiguously the presence of magnetic flux closure domains whose thickness can constitute a significant fraction $(\sim 25 \%)$ of the total film.
\end{abstract}

Keywords: magnetism; X-ray scattering; dichroism.

\section{Introduction}

Regular domain patterns are a characteristic feature of many low-dimensional systems with phases stabilized by competing interactions (Seul \& Adelman, 1995). In ultrathin magnetic films such a domain structure (Fig. 1) can occur when the electron spins favor to order perpendicular to the film plane (Hehn et al., 1996). These systems are of particular interest for magneto-optical storage devices and display the giant magneto-resistance effect utilized in magnetic field sensors (Gregg et al., 1996). It is the competition between a perpendicular magneto-crystalline anisotropy and the dipolar spin-spin interaction that leads to the regular domain pattern shown in Fig. 1. The demagnetized ground state is characterized by magnetic flux lines that are partially outside the sample. It was already predicted by Kittel (1946) half a century ago that internal flux closure (see Fig. 1) should produce a high degree of order in the magnetic domains. In spite of the important fundamental and technological implications for ultrathin films, such closure domains with a magnetization direction in the film plane are almost impossible to observe even with modern imaging techniques that are capable of achieving sufficient lateral resolution such as magnetic force microscopy (MFM), Lorentz microscopy or scanning electron microscopy with polarization analysis (SEMPA). This is due to the fact that these techniques often monitor the magnetic stray field outside the sample, as is the case for MFM, and provide hardly any information about the magnetic depth profile within the layer (Hehn et al., 1996). Lorentz microscopy, on the other hand, is performed in the transmission mode and hence averages over the whole

$\dagger$ Present address: Institut für Festkörperforschung, Forschungszentrum Jülich, 52425 Jülich, Germany. layer. In principle a surface-sensitive technique such as SEMPA could determine the existence of closure domains at the film surface (Stamm et al., 1998). However, SEMPA would be at a loss to obtain information about the depth profile.

Here we report the method of circular dichroism in $\mathrm{X}$-ray resonant magnetic scattering (CDXRMS) that can achieve all of these goals in a straightforward manner. We utilize the strong magneto-optical effects at the spin-orbit split $L_{2,3}$ absorption edges of the $3 d$ transition metals that causes a Faraday rotation of the (linearly) polarized X-rays analogous to, but much stronger than, the Kerr ellipticity in the visible region (Kortright et al., 1995). The observation of circular dichroism in the X-ray scattering signal, I, i.e. its difference between left and right circularly polarized photons, allows us to recover the phase information that is generally lost in diffraction experiments. We demonstrate that this effect can be directly related to the magnetization profile in the film.

\section{Experimental method}

Element specificity is established by tuning the X-ray energy to an absorption edge. For the transition metals studied here, such as Fe, excitation of $2 p$ electrons into unoccupied magnetically polarized $3 d$ states produces intense resonances with photon energies in the soft X-ray region $(\lambda=17.5 \AA)$. At these edges the resonant and coherent elastic absorption and emission of photons causes a strong enhancement of the magnetic scattering signal that can become comparable with the scattering from all other elemental constituents in the sample. The scattering signal measured in a diffraction experiment is the modulus square of the sum over all lattice sites, $\mathbf{r}_{n}$, of the scattering 
amplitudes, $f_{n}$, weighted by a phase factor, i.e. $I \propto\left|\sum_{n} \exp \left(i \mathbf{q} . \mathbf{r}_{n}\right) f_{n}\right|^{2}$ (q is the photon wavevector transferred in the scattering process) (Hill \& McMorrow, 1996). Hannon et al. (1998) showed that the resonant electrical dipole scattering amplitude can be written as

$$
f_{n}=\hat{\mathbf{e}}^{\prime} \cdot \hat{\mathbf{e}} F_{n}^{(0)}-i\left(\hat{\mathbf{e}}^{\prime} \times \hat{\mathbf{e}}\right) \cdot \hat{\mathbf{M}}_{n} F_{n}^{(1)}+\left(\hat{\mathbf{e}}^{\prime} \cdot \hat{\mathbf{M}}_{n}\right)\left(\hat{\mathbf{e}} \cdot \hat{\mathbf{M}}_{n}\right) F_{n}^{(2)},
$$

where $\hat{\mathbf{e}}$ and $\hat{\mathbf{e}}^{\prime}$ are the polarization vectors of the incident and scattered X-rays, respectively, and $\hat{\mathbf{M}}_{n}$ is the magnetization direction in the sample. The complex factors $F_{n}$ describe the atomic resonant excitation and decay processes. They can be expanded in terms of multipole moments of the ground state (Luo et al., 1993). While the first term in (1) is due to scattering from the Fe charge distribution, the latter two terms are purely magnetic scattering contributions. In the following we will mainly utilize the $f_{n}^{(1)}$ term to reconstruct the magnetization profile of the film. The difficulty hereby usually is that the complex factors $F_{n}$ are not very well known and can only be obtained directly under certain conditions such as for multilayered samples (Tonnerre et al., 1995; Sacchi et al., 1998). However, the case of regular domain patterns provides an elegant way of separating the three scattering contributions in (1). The lateral domain periodicity leads to purely magnetic superstructure scattering peaks located symmetrically around the specularly reflected X-ray beam. For films which are structurally well ordered and possess smooth interfaces, the charge scattering term in (1) contributes only to the specular peak. The two magnetic terms are linear and quadratic in $\hat{\mathbf{M}}_{n}$ and cause magnetic peaks at wavevectors $\pm \tau$ and $\pm 2 \tau$, respectively $(2 \pi / \tau$ is the

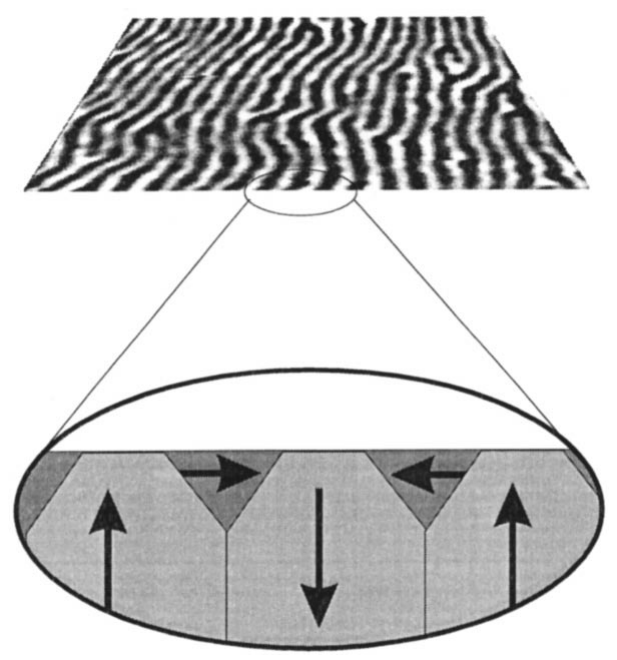

Figure 1

Magnetic force microscopy (MFM) image of a $2 \mu \mathrm{m} \times 2 \mu \mathrm{m}$ area of a $400 \AA$-thick FePd film grown epitaxically on an $\operatorname{MgO}(001)$ substrate. The contrast in the image is due to magnetic domains with flux lines directed upwards and downwards with respect to the film plane. The enlarged area shows schematically the magnetic layer profile expected from Kittel (1946) for the presence of closure domains with a magnetization direction in the film plane. domain periodicity) (Hill \& McMorrow, 1996; Hannon et al., 1998).

In order to measure both magnetic scattering contributions we used the experimental geometry shown in Fig. 2. $\mathrm{X}$-rays were incident along the stripe domains at a grazing angle $\Theta$ relative to the surface. Scattered X-rays were detected at a different $y$-position (see Fig. 2) which determined the wavevector transfer perpendicular to the stripe domains. To assess the scattering from the individual domains it is conventional to divide the light polarization into the two linear components $\sigma$ and $\pi$ that are perpendicular and parallel to the scattering plane, respectively. For the scattering geometry shown in Fig. 2 and concentrating on the second term in (1) there is more than one scattering path which is producing $\pi$-polarized scattered light (Hill \& McMorrow, 1996). For $\hat{\mathbf{M}}_{n}$ perpendicular to the film, $\sigma$-polarized incident radiation experiences a Faraday rotation producing a $\pi$ component (Kortright et al., 1995). The other channel is $\pi-\pi$ scattering leaving the incident $\pi$-polarization unchanged (Kao, 1990). It occurs when $\hat{\mathbf{M}}_{n}$ has a component perpendicular to the scattering plane, as is the case for the closure domains. These two scattering channels leading to $\pi$-polarized light can interfere with each other. Since bulk and closure domains are located at different lateral positions, the X-rays scattered from them will experience a phase shift of $90^{\circ}$ relative to each other (see Fig. 3). Unfortunately, the phase shift between the $\sigma-\pi$ and $\pi-\pi$ scattering is lost when only linearly polarized $\mathrm{X}$-rays are used. However, the phase information can be retrieved with circularly polarized radiation. This is schematically depicted in Fig. 3 which shows a projection of the experimental geometry in Fig. 2 onto the $y z$-plane. Left and right circularly polarized light is a mixture of $\sigma$ - and $\pi$-polarizations where the $\pi$ component is advanced or retarded with respect to the $\sigma$-polarization by $90^{\circ}$, respectively. As a consequence the scattering of circularly polar-

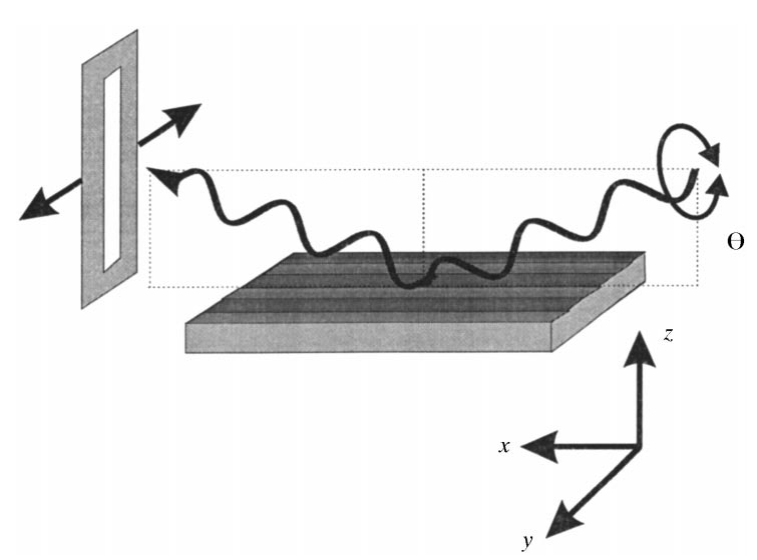

Figure 2

Schematic representation of the experimental scattering geometry. Circularly polarized X-rays are incident along the magnetic stripe domains. They are detected by a photodiode mounted behind a rectangular aperture which also selects the wavevector transferred perpendicularly to the stripes ( $y$-direction). 
ized X-rays from bulk and closure domains in Fig. 1 will result in a total relative phase shift of 0 or $180^{\circ}$ depending on the helicity, i.e. there will be either constructive or destructive interference between the two scattering channels. This is expected to result in dramatic intensity changes of the magnetic superstructure peaks with incident light helicity that is indicative for the existence of closure domains.

\section{Results and discussion}

For an experimental corroboration we used a $400 \AA$ A-thick FePd film that was grown by molecular beam epitaxy onto a $\mathrm{MgO}(001)$ substrate and capped with a $20 \AA$-thick Pd layer to prevent contamination. The growth was performed by codepositing $\mathrm{Fe}$ and $\mathrm{Pd}$ at the stoichiometric ratio. This lead to a layer-by-layer growth mode as monitored in situ by reflection high-energy electron diffraction. The sample was found by hard X-ray diffraction to be single crystalline with a chemical long-range order of $70 \%$ (Kamp et al., 1998). In Fig. 1 an MFM image of this sample is displayed in the as-grown state. It shows clearly the well ordered alternating up and down magnetic domain pattern. Almost $100 \%$ circularly polarized X-rays from beamline ID12B of the European Synchrotron Radiation Facility in Grenoble (France) were incident along the stripe domains at a grazing angle $\Theta$ that could be varied by rotating the sample around the $y$-axis in the film plane (see Fig. 2). Scattered $\mathrm{X}$-rays were detected by a photodiode mounted behind a rectangular aperture which could be scanned along the $y$-direction to select a scattering wavevector, $\mathbf{q}_{y}$, transferred perpendicular to the stripes. The entire setup was mounted in a vacuum chamber to avoid X-ray absorption by air. Typical CDXRMS scans of the scattering signal measured

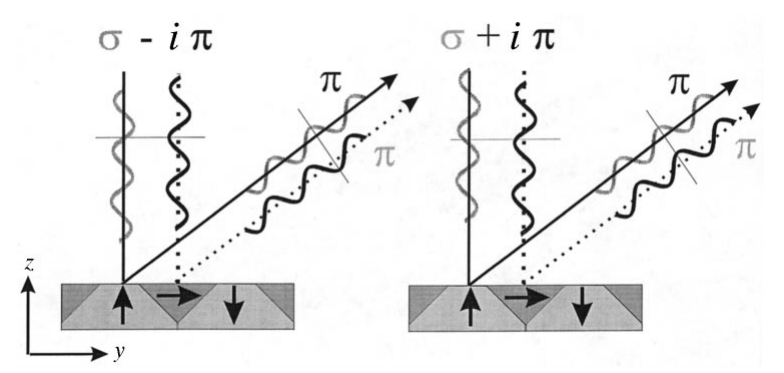

\section{Figure 3}

Projection of the experimental geometry onto the $y z$-plane (see Fig. 2). Shown are the different dominant scattering channels producing $\pi$-polarized scattered radiation for left (left panel) and right (right panel) circularly polarized X-rays (see text). For a wavevector transfer leading to the first-order magnetic satellite peaks, i.e. $\mathbf{q}_{y}= \pm \tau(2 \pi / \tau$ is the domain periodicity), the X-rays scattered from neighboring spin up or down domains experience a phase shift of $\pm 360^{\circ}$, respectively. Consequently the phase shift between $\mathrm{X}$-rays scattered from adjacent bulk and closure domains is $\pm 90^{\circ}$. The use of circularly polarized X-rays enhances this phase difference to 0 and $180^{\circ}$ (for the $+\tau$ satellite) and 180 and $0^{\circ}$ (for the $-\tau$ satellite) which is observable as interference in the scattering signal with a reversal of the X-ray helicity. over four orders of magnitude are displayed in Fig. 4. The spectra, taken with opposite light helicities at $\Theta=12^{\circ}$, show clearly the first-order (at $\left.\mathbf{q}_{y}= \pm \tau\right)$ and second-order $( \pm 2 \tau)$ magnetic peaks located symmetrically around the specularly reflected X-ray beam. We obtained $\tau=0.0069 \AA^{-1}$ that corresponded to a domain period of $909 \AA$, in good agreement with the MFM result. Fig. 4 displays clearly the strong intensity changes with light helicity in the magnetic superstructure peaks. It is also evident that the circular dichroism reverses sign for negative $\mathbf{q}_{y}$. This is expected from symmetry arguments and can be easily visualized from Fig. 3. So instead of measuring with opposite light helicities it would be sufficient to compare the magnetic peak intensities for just one spectrum but with opposite sign of $\mathbf{q}_{y}$.

In order to obtain more information about the magnetization depth profile we measured CDXRMS spectra at different incidence angles. At the chosen incidence angles the X-ray penetration depth is smaller that the film thickness and only the topmost interface is probed. Consequently the probing volume changes with incidence angle. Since the closure domains are located near the surface while the bulk domains are extended throughout the whole film, the changing probing volume will alter the effective phase difference of X-rays scattered from bulk and closure domains, thus leading to intensity oscillations of the circular dichroism signal with incidence angle. The results are collected in the inset of Fig. 4 where the ratio of the difference to sum intensities, $I_{A}=\left(I^{+}-I^{-}\right) /\left(I^{+}+I^{-}\right)$, is plotted for the first-order (solid symbols) and second-order (open symbols) magnetic peaks. Following Hill \& McMorrow (1996) we modeled the measured values taking into account all possible scattering channels according to equation (1) (lines). The results are presented in detail by

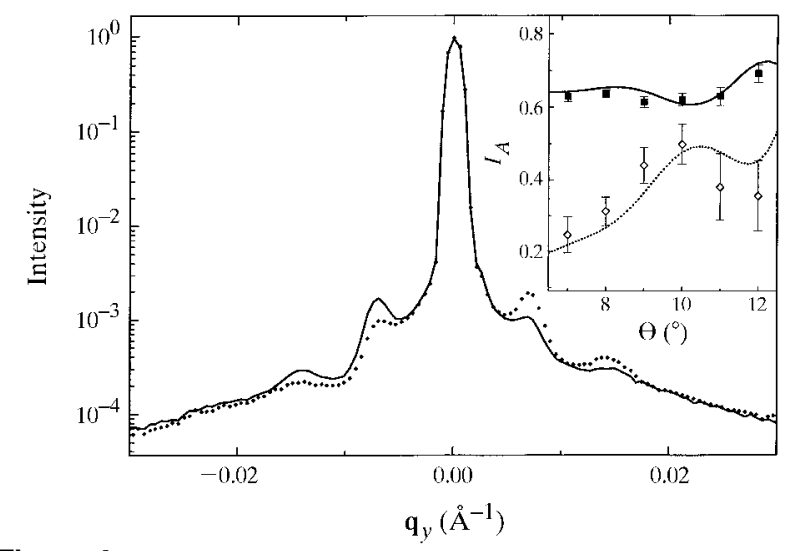

Figure 4

Diffraction scans with the wavevector, $\mathbf{q}_{y}$, transferred along the $y$ axis perpendicular to the magnetic stripes. Left (dotted line), $I^{-}$, and right (solid line), $I^{+}$, circularly polarized X-rays with energy tuned to the $\mathrm{Fe} L_{3}$ absorption edge $(\lambda=17.5 \AA)$ impinge at an angle of $\Theta=12^{\circ}$ relative to the surface plane. The inset shows the values of $I_{A}=\left(I^{+}-I^{-}\right) /\left(I^{+}+I^{-}\right)$versus $\Theta$ for the first-order (solid symbols) and second-order (open symbols) magnetic satellite peaks. The solid and dotted lines are a fit to the data as described in the text. 
Dürr et al. (1999) and we will only summarize the salient features of the model below. We assumed that closure domains were distributed uniformly over an effective depth $t$ near the surface of the film. From the specular reflectivity curve we determined the mean free X-ray path in FePd at the Fe $L_{3}$ edge to be $\sim 400 \AA$.

The $I_{A}$ signals for both magnetic satellite peaks show strong modulations with $\Theta$ which provides a direct proof of the existence of closure domains with in-plane magnetization direction. Part of the magnitude of the $I_{A}$ signals can also be due to interference between closure domains and domain walls with a magnetization direction perpendicular to the film. However, this contribution would not vary with $\Theta$. From the best fit to the data (lines in the inset of Fig. 4) we obtained an effective thickness of the closure domain layer of $t=125 \AA$, which is a significant fraction of the total film thickness. The periodic lateral modulation of the magnetization was described by Fourier transforms. Only the first-order Fourier coefficients contribute to the firstorder magnetic peaks. Here $F_{n}^{(1)}$ are mainly proportional to the magnitude of the spin magnetic moment, being its largest contribution (Luo et al., 1993). However, both firstorder and third-order Fourier coefficients are important for the second-order magnetic satellite peaks. This larger parameter set causes the fit to be overdetermined for the present limited data range. However, an extended data set obtained in destined measurements could remove this problem. The second-order magnetic peaks are interesting since the factors $F_{n}^{(2)}$ contain contributions from other ground-state moments such as the anisotropic spin-orbit coupling. These quantities are of special importance in understanding the magneto-crystalline anisotropy (van der Laan, 1999).

\section{Summary and conclusions}

We have demonstrated that the use of circular dichroism in X-ray resonant magnetic scattering can dramatically enhance our ability to determine the magnetization profiles of ultrathin films. The interference observed between purely magnetic scattering channels is a direct tool for studying closure domains and domain walls. This technique is not restricted to the case of well ordered domain structures but is expected to work also for more irregular domain distributions. The method is also easy to apply in the presence of applied magnetic fields and allows the investigation of magnetic switching phenomena (Freeland et al., 1998). Also, photon energy-dependent measurements might result in site-resolved determination of orbital and spin magnetic moments (Tonnerre et al., 1995). Finally, we note that it is also possible to observe interference between structural and magnetic scattering channels which would measure the correlated charge and magnetic roughness of interfaces (Osgood et al., 1999).

We thank K. Larsson, O. Tjernberg and N. B. Brookes for their help and technical assistance, and the ESRF staff for the excellent operational conditions.

\section{References}

Dürr, H. A., Dudzik, E., Dhesi, S. S., Goedkoop, J. B., van der Laan, G., Belakhovsky, M., Mocuta, C., Marty, A. \& Samson, Y. (1999). Science, 284, 2166-2168.

Freeland, J. W., Bussmann, K., Lubitz, P., Idzerda, Y. U. \& Kao, C.-C. (1998). Appl. Phys. Lett. 73, 2206-2208.

Gregg, J. F., Allen, W., Ounadjela, K., Viren, M., Hehn, M., Thompson, S. M. \& Coey, J. M. D. (1996). Phys. Rev. Lett. 77, 1580-1583.

Hannon, J. P., Trammell, G. T., Blume, M. \& Gibbs, D. (1988). Phys. Rev. Lett. 61, 1245-1248.

Hehn, M., Padovani, S., Ounadjela, K. \& Bucher, J. P. (1996). Phys. Rev. B, 54, 3428.

Hill, J. P. \& McMorrow, D. F. (1996). Acta Cryst. A52, 236-244.

Kamp, P., Belakhovsky, M., Boeglin, C., Hürr, H. A., van der Laan, G., Schille, P., Rogalev, A., Goulon, J., Gehanno, V., Marty, A. \& Gilles, B. (1998). Physica B, 248, 127-132.

Kao, C. (1990). Phys. Rev. Lett. 65, 3739-3742.

Kittel, C. (1946). Phys. Rev. 70, 965-968.

Kortright, J. B., Rice, M. \& Carr, R. (1995). Phys. Rev. B, 51, 10240-10243.

Laan, G. van der (1999). Phys. Rev. Lett. 82, 640-643.

Luo, J., Trammell, G. T. \& Hannon, J. P. (1993). Phys. Rev. Lett. 71, 287-290.

Osgood, R. M. III, Sinha, S. K., Freeland, J. W., Idzerda, Y. U. \& Bader, S. D. (1999). J. Appl. Phys. 85, 4619-4621.

Sacchi, M., Hague, C. F., Pasquali, L., Mirone, A., Mariot, J.-M., Isberg, P., Gullikson, E. M. \& Underwood, J. H. (1998). Phys. Rev. Lett. 81, 1521-1524.

Seul, M. \& Adelman, D. (1995). Science, 267, 476-479.

Stamm, C., Marty, F., Vaterlaus, A., Weich, V., Egger, S., Maier, U., Ramsperger, U., Fuhrmann, H. \& Pescia, D. (1998). Science, 282, 449-451.

Tonnerre, J.-M., Seve, L., Raoux, D., Soullie, G., Rodmacq, B. \& Wolfers, P. (1995). Phys. Rev. Lett. 75, 740-743. 